\title{
Aplicação clínica do alongamento muscular: breve revisão
}

\author{
Clinical application of muscle stretching: a brief review
}

Ercole C. Rubini

Paulo T. V. Farinatti

Elirez B. Silva*

\section{Resumo}

Exercícios de alongamento são usualmente recomendados em programas de treinamento físico. Nos últimos anos, os efeitos da hipomobilidade ou hipermobilidade na incidência de lesões vêm sendo investigados, bem como suas relações com doenças. O possível efeito do alongamento sobre o sistema cardiovascular também vem sendo alvo de interesse. Sendo assim, o objetivo desta breve revisão foi analisar os estudos que investigaram a aplicação clínica do alongamento muscular. Os artigos foram obtidos a partir de busca realizada nas principais bases de dados: National Library of Medicine (MEDLINE) (1966-2011), EMBASE (1974-2011), Cochrane Database of Systematic Reviews (Cochrane Reviews) (1993-2011), Literatura Latino-Americana e do Caribe em Ciências da Saúde (LILACS) (1982-2011), Physiotherapy Evidence Database (PEDro) e Scientific Eletronic Library Online (SciELO) (1997-2011); e foram originalmente publicados em língua inglesa e portuguesa. Não houve limite de data. Para cada base de dados foram utilizadas combinações de palavras-chave e seus respectivos sinônimos em inglês ou português. Verificou-se que hipomobilidade e hipermobilidade apresentam associação com problemas diversos de saúde, sendo importante analisar essas condições em avaliações físicas e funcionais. No primeiro caso, os exercícios de alongamento muscular deverão ser evitados, enquanto no segundo deveriam ser priorizados. Recentemente, estudos têm demonstrado que o alongamento muscular é capaz de produzir respostas cardiovasculares não negligenciáveis, mormente na frequência cardíaca, pressão arterial sistólica e diastólica, duplo produto e variabilidade da frequência cardíaca. Investigações nesse campo são necessárias, pois tais respostas podem ter implicações relevantes na segurança dos exercícios em determinados casos, mas também podem se associar a efeitos benéficos em longo prazo.

Descritores: Flexibilidade; Exercício; Aptidão física; Sistema cardiovascular; Saúde. 


\section{Abstract}

Stretching exercises are usually recommended within physical training programs. In the last few years, studies have focused on the relationship between hypomobility and hypermobility with the incidence of injuries and different diseases. The possible effect of muscle stretching on the cardiovascular system has been also investigated. Therefore, the aim of this brief review was to analyze studies investigating potential clinical application of muscle stretching. The articles were obtained from the following databases: National Library of Medicine (MEDLINE) (1966-2011), EMBASE (1974-2011), Cochrane Database of Systematic Reviews (Cochrane Reviews) (1993-2011), Literatura LatinoAmericana e do Caribe em Ciências da Saúde (LILACS) (1982-2011), Physiotherapy Evidence Database (PEDro) and Scientific Electronic Library Online (SciELO) (1997-2011), originally published in English and Portuguese, with no date limits. Combinations of keywords and their synonyms in English or Portuguese were used in each database. It was found that both hypomobility and hypermobility are associated with health problems. It is therefore important to assess such conditions in the context of physical and functional evaluations. In the first case muscle stretching should be avoided, whereas in the second they should be prioritized. Recently, some studies have shown that muscle stretching can induce moderate to high increases in cardiovascular responses, particularly heart rate, systolic and diastolic blood pressures, rate pressure product, and heart rate variability. Additional research on this field is needed, since such responses may compromise exercise safety in some cases, but also be related to beneficial long term adaptations.

Keywords: Flexibility; Exercise; Physical fitness; Cardiovascular system; Health.

\section{Introdução}

Tradicionalmente, os exercícios de alongamento foram sempre recomendados e incluídos em programas de exercícios físicos, seja no contexto do treinamento de atletas de alto rendimento, seja visando promoção da saúde ou reabilitação de indivíduos lesionados. ${ }^{1-4}$

Nos últimos anos, a aplicação clínica desse tipo de exercício vem sendo cada vez mais investigada, desde o que se refere às enfermidades relacionadas ao sistema musculoesquelético (no que tange à hipomobilidade ou hipermobilidade $)^{2}$ até benefícios sobre o sistema cardiovascular, ${ }^{5-16}$ abrindo amplo campo de investigação.

Sendo assim, esta breve revisão teve como objetivo analisar os estudos que investigaram as possíveis aplicações clínicas dos exercícios de alongamento.

\section{Métodos}

Os artigos foram obtidos a partir de busca realizada nas seguintes bases de dados: National Library of Medicine (MEDLINE) (1966-2011), EMBASE (1974-2011), Cochrane Database of Systematic Reviews (Cochrane Reviews) (19932011), Literatura Latino-Americana e do Caribe em Ciências da Saúde (LILACS) (1982-2011), Physiotherapy Evidence Database (PEDro) e Scientific Eletronic Library Online (SciELO) (1997-2011); e foram originalmente publicados em língua inglesa e portuguesa. Não houve limite de data. Para cada base de dados foram utilizadas combinações de palavras-chave e seus respectivos sinônimos em inglês ou português. Tais sinônimos foram obtidos após identificação nos Descritores em Ciências da Saúde (DeCS). Após, os sinônimos referentes aos vocábulos traduzidos foram identificados no Medical Subjetic Headings (MeSH).

As palavras-chave utilizadas para a busca em todas as bases de dados foram na língua portuguesa: "alongamento", "flexibilidade", "sistema cardiovascular", "frequência cardíaca”, "pressão arterial”, "hipermobilidade" e "hipomobilidade"; e na língua inglesa: stretching, flexibility, stretch, cardiovascular system, heart rate, blood pressure, hypermobility $\mathrm{e}$ hypomobility, devidamente combinadas entre si. As referências dos artigos identificados também foram consideradas para esta revisão. 


\section{Doenças associadas à hipermobilidade e à hipomobilidade}

Tanto a hipomobilidade quanto a hipermobilidade estão associadas a enfermidades e problemas de saúde. ${ }^{17-19} \mathrm{~A}$ hipermobilidade ou hiperflexibilidade pode ser de origem genética, podendo estar associada ou não, a alguma doença, como são os casos da síndrome de Marfan, síndrome de Ehler-Danlos, osteogênese imperfeita, síndrome de Larsen, síndrome de Down ${ }^{20}$ ou prolapso da válvula mitral. ${ }^{21}$

Em situações nas quais a hipermobilidade está associada à presença de dores articulares ou musculoesqueléticas, com evolução de pelo menos três meses ininterruptos, pode ser diagnosticada a síndrome da hipermobilidade que é caracterizada como doença reumatológica. ${ }^{20,22}$ Nota-se, contudo, que se pode atingir um quadro clínico de hipermobilidade após um extenso período de treinamento da flexibilidade, o que pode gerar instabilidade articular e predispor a lesões musculoarticulares. ${ }^{2}$ Essa hipermobilidade gerada pelo treinamento é comum entre os bailarinos, ${ }^{23}$ por exemplo, assim como as lesões musculoesqueléticas. ${ }^{24,25}$

A relação entre hipermobilidade e maior predisposição a lesões já é bem-estabelecida na literatura. Um estudo realizado com 675 soldados com 17 anos de idade, após dois meses de acompanhamento, constatou que a incidência de lesões musculoligamentares foi significantemente maior nos que tinham maior lassitude articular. ${ }^{26}$ Resultados similares foram observados em outro estudo realizado com 200 jogadoras de netball, relatando-se maior incidência de lesões nas atletas com hipermobilidade..$^{27}$ Portanto, ao contrário do que se observa em outras qualidades físicas, como a força e potência aeróbia, pode-se afirmar que um quadro de excesso de flexibilidade não seria desejável para a saúde - nesses casos, os exercícios de alongamento deveriam ser contraindicados.
Por outro lado, com o envelhecimento o nível de flexibilidade tende a diminuir, ${ }^{28} \mathrm{o}$ que influencia negativamente a realização de atividades da vida diária. A hipomobilidade também está associada a algumas doenças como a osteoartrite, a espondilite anquilosante, artrite reumatoide ${ }^{2}$ e em determinadas situações pode acarretar uma compressão neural, frequentemente dolorosa, como no caso das lombalgias - melhorar a flexibilidade no movimento de flexão anterior do tronco seria recomendado em tais situações. ${ }^{29}$ É muito comum, também, que após uma fratura na qual o membro ficou imobilizado ou após uma cirurgia na qual ocorreu uma restrição de movimentos aconteça uma diminuição desse arco de movimento. ${ }^{30} \mathrm{Em}$ tais quadros, a recuperação do arco de movimento de forma plena é uma prioridade e o treinamento da flexibilidade deve ser aplicado.

\section{Possíveis efeitos sobre o sistema cardiovascular}

Pouco se sabe sobre a influência dos exercícios de alongamento sobre as respostas cardiovasculares. Apesar disso, é comumente aceito que indivíduos mal-condicionados, como idosos ou cardiopatas, podem treinar flexibilidade despreocupadamente. Essa noção nasce da crença que exercícios de alongamento não exercem influência importante sobre o sistema cardiovascular. No entanto, já existem estudos que demonstraram haver alterações fisiológicas agudas importantes, podendo, em algumas situações, representar sobrecargas não negligenciáveis em termos de segurança. ${ }^{5-15}$

Nos 11 estudos presentemente localizados, os métodos de alongamento estático e facilitação neuromuscular proprioceptiva foram investigados em homens e mulheres, que realizaram de 1 a 25 séries, num tempo total de exercício que variou de 10 a 600 segundos, nos músculos flexores plantares, isquiopoplíteos, eretor da espinha e ombro, verificando o efeito desses alongamentos musculares sobre a pressão arterial, frequência cardíaca, variabilidade da 
frequência cardíaca e duplo produto, com a utilização de frequencímetro, eletrocardiograma, Finapress e mais recentemente do Finometer. ${ }^{5-15}$

Os resultados obtidos por esses estudos indicam que podem ocorrer aumentos agudos na variabilidade da frequência cardíaca, pressão arterial, frequência cardíaca e, em consequência dos dois últimos, do duplo produto, em função de diferentes tipos de treinamento da flexibilidade (como os métodos estático e facilitação neuromuscular proprioceptiva). Isso pode se tornar mais relevante quando se tratam de indivíduos idosos, hipertensos ou com alguma cardiopatia. No entanto, a magnitude dessas alterações precisa ser mais profundamente investigada e entendida.

Por outro lado, encontra-se na literatura, um único estudo crônico, no qual 15 atletas de bodybuilding do sexo masculino, que treinavam pelo menos há um ano duas horas por dia, cinco vezes por semana e que relataram ter flexibilidade limitada, submeteram-se a 28 dias de treinamento sem supervisão, durante 15 minutos. ${ }^{16}$ Neste estudo, os resultados demonstraram que além do aumento da flexibilidade houve um aumento da variabilidade da frequência cardíaca como adaptação a um programa de exercícios de alongamento, o que representa um benefício importante para a saúde cardiovascular. ${ }^{16}$

Confirmados esses achados, os exercícios de alongamento poderão ser parte importante não só da prevenção, mas também do tratamento de algumas doenças cardiovasculares.

\section{Conclusão}

$\mathrm{Na}$ última década vários estudos foram publicados sobre alongamento e flexibilidade e muitos mitos foram esclarecidos. Atualmente sabe-se que a flexibilidade é uma capacidade motora que exige um nível ótimo, ou seja, não é bom ter muita nem pouca. Isso a diferencia das outras capacidades motoras nas quais quanto mais desenvolvidas melhor. Ter hipomobilidade ou hipermobilidade está diretamente associado a dificuldades de realização de tarefas cotidianas, bem como a uma maior probabilidade de lesão osteomioarticular.

Portanto, uma boa prescrição depende necessariamente de uma boa avaliação. Caso se constate a hipomobilidade, o exercício de alongamento muscular passa a ser uma das prioridades da sessão de exercício, enquanto que no caso da existência de uma hipermobilidade, os exercícios de alongamento muscular devem ser contraindicados.

Estudos recentes também já verificaram alguns efeitos do alongamento muscular sobre as respostas cardiovasculares e isso talvez também passe a ter uma grande relevância clínica, principalmente em se tratando de indivíduos mais velhos, hipertensos ou cardiopatas. Desta forma, a relevância clínica de se conhecer e aplicar exercícios de alongamento muscular é muito importante, podendo fazer parte tanto de um programa preventivo ou terapêutico em relação a determinadas lesões ou doenças.

\section{Referências}

1. American College of Sports Medicine. The recommended quantity and quality of exercise for developing and maintaining cardiorespiratory and muscular fitness, and flexibility in healthy adults. Position Stand. Med Sci Sports Exerc. 2011;43(7):1334-59.

2. Rubini EC. Treinamento de flexibilidade: da teoria à prática. $1^{a}$. ed. Rio de Janeiro: Ed. Sprint; 2010. 112 p.

3. Herbert RD, Gabriel M. Effects of stretching before and after exercising on muscle soreness and risk of injury: systematic review. BMJ. 2002;325(7362):468. http://dx.doi. org/10.1136/bmj.325.7362.468

4. Rubini EC, Costa ALL, Gomes PSC. Effects of stretching on strength performance. Sports Med. 2007;37(3):213-24. http://dx.doi. org/10.2165/00007256-200737030-00003

5. Holt LE, Pelham TW, Campagna PD. Hemodynamics during a machine-aided flexibility protocol. Can J Applied Physiol. 1995;20(4):407-16.

6. Cornelius WL, Jensen RL, Odell ME. Effects of PNF stretching phases on acute arterial blood pressure. Can J Applied Physiol. 1995;20(2):222-9.

7. Baum K, Selle K, Leyk D, Essfeld D. Comparison of blood pressure and heart rate responses to isometric exercise and passive muscle stretch in humans. Eur J Appl Physiol 
Occup Physiol. 1995;70(3):240-5. http://dx.doi. org/10.1007/BF00238570

8. Gladwell VF, Coote JH. Heart rate at the onset of muscle contraction and during passive muscle stretch in humans: a role for mechanoreceptors. J Physiol. 2002;540:1095-102. http://dx.doi.org/10.1113/ jphysiol.2001.013486

9. Tokizawa K, Mizuno M, Nakamura Y, Muraoka I. Passive triceps surae stretch inhibits vasoconstriction in the nonexercised limb during posthandgrip muscle ischemia. J Appl Physiol. 2004;97(5):1681-5. http://dx.doi. org/10.1152/japplphysiol.00312.2004

10. Fisher JP, Bell MP, White MJ. Cardiovascular responses to human calf muscle stretch during varying levels of muscle metaboreflex activation. Exp Physiol. 2005;90(5):773-81. http://dx.doi.org/10.1113/ expphysiol.2005.030577.

11. Gültekin Z, Kin-isler A, Sürenkök Ö. Hemodynamic and lactic acid responses to proprioceptive neuromuscular facilitation exercise. J Sports Sci Med. 2006;5:375-80.

12. Cui J, Blaha C, Moradkhan R, Gray KS, Sinoway LI. Muscle sympathetic nerve activity responses to dynamic passive muscle stretch in humans. J Physiol. 2006;576(2):625-34. http://dx.doi.org/10.1113/ jphysiol.2006.116640

13. Drew RC, Bell MP, White MJ. Modulation of spontaneous baroreflex control of heart rate and indexes of vagal tone by passive calf muscle stretch during graded metaboreflex activation in humans. J Appl Physiol. 2008;104:716-23. http://dx.doi.org/10.1152/ japplphysiol.00956

14. Farinatti PTV, Brandão C, Soares PPS, Duarte AF. Acute effects of stretching exercise on the heart rate variability in subjects with lower flexibility levels. J Strength Cond Res. 2011;25(6):1579-85. http://dx.doi.org/10.1519/ JSC.0b013e3181e06ce1

15. Farinatti PTV, Soares PPS, Monteiro WD, Duarte AF, Castro LA. Cardiovascular responses to passive static flexibility exercises are influenced by the stretched muscle mass and valsalva maneuver. Clinics. 2011;66(3):459-64. http://dx.doi.org/10.1590/ S1807-59322011000300017

16. Mueck-Weymann M, Janshoff G, Mueck H. Stretching increases heart rate variability in healthy athletes complaining about limited muscular flexibility. Clin Auton Res. 2004;14(1):15-8. http://dx.doi.org/10.1007/ s10286-004-0123-0

17. Grahane R. Time to take hypermobility seriously (in adults and children). Rheumatology. 2001;40(5):485-7. http://dx.doi. org/10.1093/rheumatology/40.5.485

18. Araújo CGS, Chaves CPG. Prolapso da Valva
Mitral em Mulheres Adultas: características clínicas, fisiológicas e cineantropométricas. Rev SOCERJ. 2007;20(2):112-20.

19. Grahane R. Joint hypermobility and genetic collagen disorders: are they related? Arch Dis Child. 1999;80(2):188-91. http://dx.doi. org/10.1136/adc.80.2.188

20. McCormack M, Briggs J, Hakim A, Grahame R. Joint laxity and the benign joint hypermobility syndrome in student and professional ballet dancers. J Rheumatol. 2004;31(1):173-8.

21. Byhring S, Bø K. Musculoskeletal injuries in the Norwegian National Ballet: a prospective cohort study. Scand J Med Sci Sports. 2002;12(6):365-70. http://dx.doi.org/10.1034/ j.1600-0838.2002.01262.x

22. Hincapié CA, Morton EJ, Cassidy JD. Musculoskeletal injuries and pain in dancers: a systematic review. Arch Phys Med Rehabil. 2008;89(9):1819-29. http://dx.doi.org/10.1016/j. apmr.2008.02.020

23. Acasuso-Díaz M, Collantes Estévez E, Sánchez Guijo P. Joint hyperlaxity and musculoligamentous lesions: study of a population of homogeneous age, sex and physical exertion. Br J Rheumatol. 1993;32(2):120-2. http://dx.doi.org/10.1093/ rheumatology/32.2.120

24. Smith R, Damodoran AK, Swaminathan S, Campbell R, Barnsley L. Hypermobility and sports injury in junior netball players. Br J Sports Med. 2005;39(9):628-31. http://dx.doi. org/10.1136/bjsm.2004.015271

25. Gajdosik RL, Vander LDW, Williams AK. Influence of age on length and passive elastic stiffness characteristics of the calf muscle-tendon unit of women. Phys Ther. 1999;79(9):827-38.

26. Polito MD, Neto GAM, Lira VA. Componentes da aptidão física e sua influência sobre a prevalência de lombalgia. Rev Bras Ciênc Mov. 2003;11(2):35-40.

27. Williams PE, Goldspink G. Connective tissue changes in immobilised muscle. J Anat. 1984;138(2):343-50.

28. Decoster LC, Vailas JC, Lindsay RH, Williams GR. Prevalence and features of joint hypermobility among adolescents athletes. Arch Pediatr Adolesc Med. 1997;151(10):98992.

29. Perrini F, Tallents RH, Katzberg RW, Ribeiro RF, Kyrkanides S, Moss ME. Generalized joint laxity and temporomandibular disorders. J Orofac Pain. 1997;11(3):215-21.

30. Martin-Santos R, Bulbena A, Porta M, Gago J, Molina L, Duró JC. Association between joint hypermobility syndrome and panic disorder. Am J Psychiatry. 1998;155(11):1578-83. http:// dx.doi.org/10.1176/appi.psy.51.1.55. 


\section{Autores}

\section{Ada Fernanda P. S. Lima}

Laboratório de Atividade Física e Promoção da Saúde. Instituto de Educação Física e Desportos. Universidade do Estado do Rio de Janeiro. Rio de Janeiro, RJ, Brasil.

\section{Alexandre H. Okano}

Departamento de Educação Física. Centro de Ciências da Saúde. Universidade Federal do Rio Grande do Norte. Natal, RN, Brasil.

\section{Alice R. Sampaio}

Laboratório de Atividade Física e Promoção da Saúde. Instituto de Educação Física e Desportos. Universidade do Estado do Rio de Janeiro. Rio de Janeiro, RJ, Brasil.

\section{Ana Paula M. Guttierres}

Laboratório de Atividade Física e Promoção da Saúde. Instituto de Educação Física e Desportos. Universidade do Estado do Rio de Janeiro. Rio de Janeiro, RJ, Brasil.

\section{Astrogildo V. Oliveira Júnior}

Departamento de Educação Física e Folclore. Colégio Pedro II. Rio de Janeiro, RJ, Brasil.

\section{Brenno S. Silva}

Programa de Pós-graduação em Ciências da Atividade Física. Universidade Salgado de Oliveira. Niterói, RJ, Brasil.

\section{Daniel A. Bottino}

Laboratório de Pesquisas Clínicas e Experimentais em Biologia Vascular. Centro Biomédico.

Universidade do Estado do Rio de Janeiro. Rio de Janeiro, RJ, Brasil.

\section{Dionizio Mendes Ramos Filho}

Laboratório de Bioenergética e Fisiologia Mitocondrial. Centro de Ciências da Saúde. Universidade Federal do Rio de Janeiro. Rio de Janeiro, RJ, Brasil.

\section{Elirez B. Silva}

Laboratório de Pesquisa Clínica Escola. Departamento de Fisioterapia. Universidade Gama Filho. Rio de Janeiro, RJ, Brasil.

\section{Ercole C. Rubini}

Laboratório de Fisiologia do Exercício. Universidade Estácio de Sá. Rio de Janeiro, RJ, Brasil.

\section{Fabrício V. A. Vasconcellos}

Centro de Investigação, Formação, Inovação, Intervenção e Desporto. Faculdade de Desporto. Universidade do Porto. Porto, Portugal.

\section{Felipe A. da Cunha}

Programa de Pós-graduação em Ciências Médicas. Universidade do Estado do Rio de Janeiro. Rio de Janeiro, RJ, Brasil.

\section{Fernanda Monteiro}

Laboratório de Atividade Física e Promoção da Saúde. Instituto de Educação Física e Desportos. Universidade do Estado do Rio de Janeiro. Rio de Janeiro, RJ, Brasil.

\section{Flávia Porto}

Programa de Pós-graduação em Ciências do Exercício e do Esporte. Universidade Gama Filho. Rio de Janeiro, RJ, Brasil. 


\section{Gustavo C. Lopes}

Laboratório de Atividade Física e Promoção da Saúde. Instituto de Educação Física e Desportos. Universidade do Estado do Rio de Janeiro. Rio de Janeiro, RJ, Brasil.

\section{Jonas L. Gurgel}

Programa de Pós-graduação em Ciências

Cardiovasculares. Universidade Federal Fluminense.

Niterói, RJ, Brasil.

\section{Jonathan Myers}

VA Palo Alto Health Care System. Cardiology Division. Stanford University. Palo Alto, California, United States.

\section{Karynne Grutter}

Laboratório de Anatomia Humana. Universidade Castelo Branco. Rio de Janeiro, RJ, Brasil.

\section{Lenifran Matos-Santos}

Programa de Pós-graduação em Ciências da Atividade Física. Universidade Salgado de Oliveira. Niterói, RJ, Brasil.

\section{Lorena Paes}

Programa de Pós-graduação em Fisiopatologia Clínica e Experimental. Universidade do Estado do Rio de Janeiro. Rio de Janeiro, RJ, Brasil.

\section{Luciane P. da Costa}

Laboratório de Atividade Física e Promoção da Saúde. Instituto de Educação Física e Desportos. Universidade do Estado do Rio de Janeiro. Rio de Janeiro, RJ, Brasil.

\section{Luiz G. Kraemer-Aguiar}

Departamento de Medicina Interna. Faculdade de Ciências Médicas. Universidade do Estado do Rio de Janeiro. Rio de Janeiro, RJ, Brasil.

\section{Matheus R. Hausen}

Laboratório de Atividade Física e Promoção da Saúde. Instituto de Educação Física e Desportos. Universidade do Estado do Rio de Janeiro. Rio de Janeiro, RJ, Brasil.

\section{Paulo T. V. Farinatti}

Laboratório de Atividade Física e Promoção da Saúde. Instituto de Educação Física e Desportos. Universidade do Estado do Rio de Janeiro. Rio de Janeiro, RJ, Brasil.

\section{Rafael A. Montenegro}

Programa de Pós-graduação em Fisiopatologia Clínica e Experimental. Universidade do Estado do Rio de Janeiro. Rio de Janeiro, RJ, Brasil.

\section{Raul A. Freire}

Programa de Pós-graduação em Ciências da Atividade Física. Universidade Salgado de Oliveira. Niterói, RJ, Brasil.

\section{Renato O. Massaferri}

Programa de Pós-graduação em Ciências da Atividade Física. Universidade Salgado de Oliveira. Niterói, RJ, Brasil.

\section{Ricardo B. Oliveira}

Laboratório de Atividade Física e Promoção da Saúde. Instituto de Educação Física e Desportos. Universidade do Estado do Rio de Janeiro. Rio de Janeiro, RJ, Brasil.

\section{Ricardo G. Cordeiro}

Programa de Pós-graduação em Ciências da Atividade Física. Universidade Salgado de Oliveira. Niterói, RJ, Brasil.

\section{Sérgio Machado}

Programa de Pós-graduação em Ciências da Atividade Física. Universidade Salgado de Oliveira. Niterói, RJ, Brasil.

\section{Taciana Pinto}

Laboratório de Atividade Física e Promoção da Saúde. Instituto de Educação Física e Desportos. Universidade do Estado do Rio de Janeiro. Rio de Janeiro, RJ, Brasil.

\section{Tainah P. Lima Monteiro}

Programa de Pós-graduação em Ciências Médicas. Faculdade de Ciências Médicas. Universidade do Estado do Rio de Janeiro. Rio de Janeiro, RJ, Brasil.

\section{Tânia M. P. F. Paschoalino}

Hospital Universitário Antônio Pedro. Universidade Federal Fluminense. Niterói, RJ, Brasil.

\section{Walace D. Monteiro}

Laboratório de Atividade Física e Promoção da Saúde. Instituto de Educação Física e Desportos. Universidade do Estado do Rio de Janeiro. Rio de Janeiro, RJ, Brasil. 